Théologiques

Théologiques

\title{
Toucher Jésus le Ressuscité
}

\section{Marie de Magdala et Thomas le Jumeau en Jean 20}

\section{Sandra M. Schneiders}

Volume 15, numéro 2, 2007

La traduction des textes sacrés

URI : https://id.erudit.org/iderudit/017777ar

DOI : https://doi.org/10.7202/017777ar

Aller au sommaire du numéro

\section{Éditeur(s)}

Faculté de théologie et de sciences des religions, Université de Montréal

ISSN

1188-7109 (imprimé)

1492-1413 (numérique)

Découvrir la revue

Citer cet article

Schneiders, S. M. (2007). Toucher Jésus le Ressuscité : marie de Magdala et Thomas le Jumeau en Jean 20. Théologiques, 15(2), 163-192.

https://doi.org/10.7202/017777ar
Résumé de l'article

Cet article s'intéresse au rôle que joue la corporéité de Jésus dans sa relation avec les disciples après la résurrection. À travers l'étude de Jn 20, notamment les commandements contradictoires que Jésus adresse à Marie de Magdala (20,11-18) et à Thomas (20,24-29), il propose de comprendre que la résurrection n'est pas physique mais qu'elle est corporelle. Le corps du Ressuscité continuerait ainsi de médiatiser sa relation aux disciples, d'une façon qui est à la fois en continuité et en discontinuité avec la manière dont il le faisait durant sa carrière prépascale.
Tous droits réservés ( Faculté de théologie et de sciences des religions, Université de Montréal, 2007
Ce document est protégé par la loi sur le droit d'auteur. L'utilisation des services d'Érudit (y compris la reproduction) est assujettie à sa politique d'utilisation que vous pouvez consulter en ligne.

https://apropos.erudit.org/fr/usagers/politique-dutilisation/ 


\title{
Toucher Jésus le Ressuscité
}

\section{Marie de Magdala et Thomas le Jumeau en Jean $20^{1}$}

\author{
Sandra M. SCHNEIDERS \\ Jesuit School of Theology \\ Berkeley, CA
}

\section{Introduction}

En dernière analyse, tout ce qu'affirme la théologie à propos de la résurrection du corps de ceux qui sont morts en Christ est validé ou invalidé par ce que nous disons au sujet de la résurrection corporelle de Jésus luimême. Or, notre seul accès à la résurrection de Jésus - que celle-ci soit comprise corporellement, physiquement, spirituellement ou d'aucune de ces manières - est le Nouveau Testament. Bien qu'il y ait beaucoup de matériel dans le Nouveau Testament au sujet de la résurrection, seulement trois passages traitent directement de la corporéité du Ressuscité. Le premier se trouve en 1Co 15 : Paul y discute avec les membres de la communauté corinthienne qui, pour des raisons philosophiques, considéraient la matérialité en général, et le corps en particulier, comme sans valeur. Paul argumente en faveur de la possibilité, de la réalité et de la signifiance de la résurrection corporelle comme telle, que ce soit pour Jésus ou pour les croyants $^{2}$. Le deuxième texte est Lc 24,36-43, un récit de l'apparition de Jésus aux disciples, surpris et terrifiés, qui pensent voir un fantôme. Le Ressuscité les invite à vérifier, tactilement, la solidité de son corps, "chair et os ", afin de les convaincre qu'il est bien le Jésus qui a été crucifié et non

1. Traduit de l'original anglais «Touching the Risen Jesus. Mary Magdalene and Thomas the Twin in John 20 ", Proceedings of the Catholic Theological Society of America, 60 (2005) p. 13-35, par Danielle Jodoin et Alain Gignac. À l'occasion de la réception d'un doctorat d'honneur de l'Université de Montréal, la Professeure Schneiders a présenté une conférence publique sur ce thème, le 18 octobre 2007.

2. Pour des références sur le probable Sitz im Leben idéologique de la Lettre aux Corinthiens, voir Murphy-O’Connor 1990, 812. Pour un traitement bref et excellent de la position de Paul en contraste avec la position corinthienne, voir P. Lampe (2002). 
une apparition spectrale ${ }^{3}$. Les textes de Paul et de Luc s'adressaient tous deux à des auditoires majoritairement hellénistiques, dont l'anthropologie dualiste rendait la résurrection physique a priori impossible ou sans signification, ou les deux.

Bien que ces polémiques soient intéressantes et non sans lien avec les débats contemporains sur la possibilité d'une vie après la mort, aucun de ces textes ne concerne la préoccupation première du présent article, à savoir : quel rôle la corporéité de Jésus ressuscité joue-t-elle dans la relation de Jésus avec ses disciples après la résurrection, et de quelle manière joue-t-elle ce rôle ? Est-ce que le corps de Jésus, qui médiatisait avant Pâques sa relation avec ses disciples, continue à jouer ce rôle pour ses disciples postpascals, y compris nous ${ }^{4}$ ? $\mathrm{Si}$ «corporel» est synonyme de "physique ", nous nous trouvons entre deux positions également inacceptables. Ou bien, d'une part, Jésus est simplement ressuscité (resuscitated), c'est-à-dire réanimé physiquement, ce qui le laisse toujours et nous avec lui sujets aux conditions de l'espace, du temps et de la causalité, et vulnérables face à la mort. Ou bien, d'autre part, sa résurrection est purement spirituelle et nous sommes en dehors de la foi de la communauté chrétienne en la "résurrection du corps ». De façon proleptique, je propose que la résurrection de Jésus n'est pas physique, mais est corporelle et, donc, que son corps continue à médiatiser sa relation avec ses disciples, mais d'une manière qui est à la fois en continuité et en discontinuité avec la manière dont il le faisait durant sa carrière prépascale.

Je démontrerai ceci sur la base du troisième texte du Nouveau Testament qui traite du corps de Jésus ressuscité, à savoir le récit johannique de la résurrection, Jean 20, et plus spécifiquement les versets 11 à 18 (l'épisode de Marie de Magdala) et 24 à 29 (l'épisode de Thomas le Jumeau). Dans le premier épisode, Jésus ressuscité interdit à Marie de Magdala de le toucher et, dans le second, il invite, et même il ordonne à Thomas le Jumeau de le toucher. Notre question est donc: qu'est-ce que Jean 20 dit au sujet du corps de Jésus ressuscité et de son rôle dans sa relation avec ses disciples?

3. H.-J. Eckstein (2002) discute à la fois la polémique de Luc contre le dualisme anthropologique de sa communauté à prédominance gréco-romaine et la subtile solution de remplacement à cette position proposée par l'évangéliste.

4. Suivant M. Borg (1994, 15-17), je préfère utiliser les termes «prépascal » et «postpascal » pour désigner respectivement la vie de Jésus avant et après la résurrection. Le terme traditionnel "Jésus terrestre » peut établir une dichotomie théologiquement problématique entre le «Jésus de l'histoire » et le «Christ de la foi », et implicitement nier la présence continue de Jésus ressuscité dans l'expérience historique de ses disciples terrestres contemporains. 


\section{Présuppositions au sujet de la théologie de Jean}

Avant de nous tourner vers les textes en question, nous devons poser certains fondements bibliques, d'abord quant à la théologie du quatrième évangile relative à la résurrection, et ensuite quant à la structure et à la dynamique du récit johannique de la résurrection, considéré dans sa globalité. Dans un premier temps, nous avons donc besoin de regarder brièvement l'eschatologie et l'anthropologie du quatrième évangile qui gouvernent conjointement l'approche johannique de la résurrection de Jésus. Dans un second temps, nous examinerons la progression théologique au sein du récit de résurrection johannique, ainsi que le développement de la dialectique entre voir, entendre et toucher, c'est-à-dire entre l'expérience sensorielle et la croyance.

\subsection{L'eschatologie de Jean ${ }^{5}$}

Comme il est généralement reconnu, l'eschatologie du quatrième évangile diffère de façon saisissante de celle des évangiles synoptiques. Cette différence est souvent exprimée en termes de contraste entre le jugement réalisé (c.-à-d. présent) et le jugement différé (c.-à-d. futur). Cependant, ces deux eschatologies impliquent beaucoup plus qu'une différence temporelle. Chacune est une approche théologique de la vie, de la mort, du jugement et de "l'après-vie".

Dans le quatrième évangile, la mort de Jésus n'est pas présentée comme dans les synoptiques, comme une kénose, soit le point le plus bas de sa vie terrestre, une condamnation humaine que Dieu a renversée en lui rendant justice, par la résurrection. En Jean, la mort de Jésus est l'apothéose, la culmination victorieuse de sa vie. Dans et par sa mort, Jésus est glorifié par Dieu et exalté en présence de Dieu 6 . Il est proclamé roi et il règne glorieusement sur la croix (Jn 19,19). En conséquence, Bultmann observait au milieu du XXe siècle: "If Jesus' death on the cross is already his exaltation

5. Suivant la convention parmi les chercheurs johanniques, j'emploierai le terme "Jean» pour me référer au quatrième évangéliste ou à l'évangile lui-même. Ceci n'implique aucune position sur les questions très discutées de l'authenticité johannique et de l'identité de l'évangéliste (voir Schneiders 2003, 233-254).

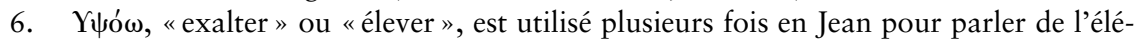
vation de Jésus sur la croix en termes d'exaltation (ex. : 3,$14 ; 8,28 ; 12,32)$; $\delta$ o $\xi \alpha \dot{\alpha} \omega$, "glorifier », est utilisé pour parler de l'effet sur Jésus de cette "élévation » dans la crucifixion (ex. : 7,$39 ; 12,16 ; 12,23 ; 13,31-32)$, nommément qu'il a été glorifié par Dieu et qu'il glorifie Dieu par sa mort. 
and glorification, his resurrection cannot be an event of special significance. No resurrection is needed to destroy the triumph which death might be supposed to have gained in the crucifixion. » (Bultmann 1955, 56)

Bultmann et d'autres ont suggéré que l'évangile de Jean se termine vraiment avec la crucifixion au chapitre 19 et que le récit de résurrection johannique est simplement une concession faite à la tradition qui était normative au moment où cet évangile a été écrit, à la fin du I $^{\mathrm{er}}$ siècle. Bien que cette conclusion soit loin d'être satisfaisante, elle soulève de façon tranchante la question du rôle joué par le récit de la résurrection dans l'évangile de Jean. Clairement, il ne joue pas le rôle de légitimation qu'il a dans les synoptiques, mais j'affirmerais qu'il s'avère crucial quant aux objectifs théologiques de Jean.

Les deux courants ou types d'eschatologie que nous trouvons dans le Nouveau Testament sont dérivés de deux types de réflexion eschatologique qui se sont développés dans les écrits tardifs de l'Ancien Testament et dans les écrits intertestamentaires ${ }^{7}$. Le premier type, que nous pourrions appeler «eschatologie de la résurrection », est futuriste et apocalyptique. Il s'est développé dans le contexte des persécutions séleucides et des polémiques maccabéennes et hasmonéennes en Palestine, aux II et $\mathrm{I}^{\mathrm{er}}$ siècles av. J.-C. Des Juifs fidèles, comme la mère et les sept frères de $2 \mathrm{M} 7$, étaient persécutés et même martyrisés pour leur fidélité à la Torah, mais ils ont été fortifiés par l'espoir qu'ils seraient justifiés par Dieu après la mort. L'expression la plus claire de cette eschatologie dans l'Ancien Testament se trouve en $2 \mathrm{M} 7$ et en Dn 12,1-3, qui prédisent le réveil de «beaucoup... qui sommeillent dans la poussière ", les deux textes étant influencés par l'image du Serviteur souffrant chez le Deutéro-Isaïe ${ }^{8}$. Les martyrs sont assurés qu'ils seront restaurés même dans leur corps, qu'Israël sera reconstitué et que l'injuste sera finalement puni.

Ce type d'eschatologie, caractéristique des évangiles synoptiques, était celui des pharisiens du temps de Jésus. Matthieu 25,31-46 dépeint un tel événement justificatif final, un jugement dernier, conçu comme cataclysme

7. Bien que la catégorie « intertestamentaire » elle-même, ainsi que les dates de la période couverte par ce terme et sa littérature soient discutées, je l'utiliserai pour désigner le chevauchement des expériences de l'Ancien Testament et du Nouveau Testament et les écrits reflétant cette expérience, s'étendant approximativement de 200 av. J.-C. à 100 apr. J.-C. Mes remerciements à mon collègue vétérotestamentaire John Endres pour son aide sur cette question.

8. Pour une comparaison détaillée des textes montrant l'influence d'Is 26,$20 ; 26,19$; 66,24 sur Dn 12,1-3, voir Schneiders 1983, 35-86. 
cosmique soudain (voir Mt 24,15-44; Mc 13 ; Lc 17,22-37), quand tous se lèveront pour comparaitre devant le Christ glorifié qui les assignera à la récompense ou à la punition éternelles sur la base de leur comportement dans cette vie. Le rôle de la résurrection corporelle, voire physique dans cette eschatologie est essentiellement fonctionnel. Elle rend le juste et l'impie présents pour un jugement final par lequel la justice divine sera entièrement manifestée. Et elle assure la participation de la personne entière, pour la sentence finale.

L'évangile de Jean, à la différence des synoptiques, fonctionne à l'intérieur de l'autre courant d'eschatologie juive tardive préchrétienne, que j'appellerai "eschatologie d'immortalité ». Cette eschatologie est réalisée et sapientielle plutôt que futuriste et apocalyptique. Elle s'est développée dans le contexte hellénistique du judaïsme de la diaspora, probablement vers la fin du II $^{\mathrm{e}}$ ou le début du $\mathrm{I}^{\mathrm{er}}$ siècle av. J.-C. Les Juifs qui étaient restés fidèles à la Torah, quoique vivants dans un contexte hellénistique, étaient persécutés et même tués non seulement par des païens, mais aussi par leurs camarades juifs assimilés culturellement et religieusement ${ }^{9}$. À nouveau, on fait appel à une solution postmortelle pour régler le problème de la victoire de l'injuste dans l'histoire. La plus claire expression (deutéro) canonique de cette eschatologie se trouve dans le livre de la Sagesse de Salomon, aux chapitres 1 à 6 .

Dans cette histoire, le héros guidé par la Sagesse, qui représente les Juifs fidèles, est persécuté à mort par les disciples de la Folie. Ceux-ci raillent sa fidélité à la Loi, nient sa revendication d'être le fils de Dieu et sont furieux d'avoir été accusés par lui d'infidélité à leur formation et à leur tradition ${ }^{10}$ (voir Sg 2,10-20). La version de la Septante du quatrième chant du Serviteur souffrant (Is 52,13 - 53,12) et de la figure du Fils de l'Homme de Dn 7 a sûrement influencé le portrait du héros martyr dans la Sagesse de Salomon ${ }^{11}$, qui a également été influencé par des notions hellénistiques d'immortalité. À la différence de l'eschatologie de résurrection

9. Pour un excellent traitement, suggestif et un brin provocateur, du livre de la Sagesse de Salomon, de son eschatologie par rapport à son Sitz im Leben et de sa relation possible au Nouveau Testament, voir Green (2003).

10. Pour le développement du genre littéraire extrabiblique du «conte de sagesse » où nous rencontrons des «héros de sagesse » en contexte non-canonique qui ressemblent à Joseph, à Daniel et à Suzanne, voir Nicholsburg 1972, 49-55. Les particularités des héros bibliques de la Sagesse sont que leur sagesse consiste en la fidélité à la Torah, plutôt qu'à un savoir-faire séculaire ou à une éthique fondée philosophiquement.

11. Voir Schneiders 1983, 98-101, pour l'évidence textuelle de cette position. 
future et apocalyptique de 2 Maccabées, cette eschatologie sapientielle présente la mort du juste comme une exaltation en vue du jugement sur ses ennemis et une entrée immédiate dans une relation intime avec Dieu, dans un royaume non-terrestre, au-delà de la mort. Bien que le héros ait été physiquement tué, le texte nous indique que "les âmes des justes sont dans la main de Dieu [...]. Aux yeux des insensés, ils ont paru mourir [...], mais les justes sont dans la paix [...]. Dieu les a mis à l'épreuve» $\left(\right.$ Sg 3,1-6 ${ }^{12}$.

La résurrection corporelle et encore moins la résurrection physique, telles que nous les voyons dans l'eschatologie résurrectionnelle, ne figurent pas explicitement dans cette compréhension sapientielle du destin du juste et de l'injuste, parce que le jugement des impies a lieu dans leur choix même du mal par lequel ils «appellent la mort » $(\mathrm{Sg} 1,16)$; les justes, quant à eux, sont exaltés par Dieu et assumés en lui au moment même de leur destruction par les impies. Cependant, l'exaltation des justes n'est pas simplement une immortalité de l'âme dans le sens philosophique grec, c'est-à-dire l'indestructibilité naturelle d'une substance spirituelle. Leur immortalité est vie dans le sens juif, c'est-à-dire un don de Dieu, qui seul la possède par nature ${ }^{13}$ et qui l'accorde librement à ceux qui sont loyaux à l'alliance. En outre, même après la mort, une vie à laquelle le corps ne participerait pas d'une manière quelconque aurait été inconcevable pour l'imaginaire juif. Ainsi, alors qu'elle n'affirme rien d'explicite au sujet de la résurrection corporelle, l'eschatologie sapientielle lui est fondamentalement compatible ${ }^{14}$ et même, d'une certaine manière, la suppose.

Cette eschatologie sapientielle est facilement discernable dans le quatrième évangile et est opératoire dans la présentation par Jean de la mort de Jésus en tant que héros de la Sagesse. Sa mort est son exaltation, dans laquelle et par laquelle ses persécuteurs sont jugés et lui, glorifié (voir Jn 16,8-11). Ceci conduit à deux conclusions à propos du rôle du récit de résurrection dans l'évangile de Jean:

12. NdT: les citations bibliques sont tirées de la Bible de Jérusalem (1998).

13. Ceci a été bien expliqué par J. Moingt (1972), qui prend essentiellement la même position que O. Cullmann en son texte classique (1955-1956).

14. Bien que cela soit au-delà de la portée de cet essai, il est important de noter que l'anthropologie juive a été influencée par la philosophie hellénistique durant la période qui précède immédiatement l'apparition du christianisme. Ceci est évident dans l'utilisation de termes tels «incorruptibilité » $(\dot{\alpha} \phi \theta \alpha \rho \sigma i ́ \alpha)$ en Sg 2,23 et «immortalité » ( $\alpha \theta \alpha \nu \alpha \sigma i \alpha)$ en $S g$ 3,4. D'autre part, l'approche typiquement biblique apparaît dans le livre de la Sagesse de Salomon dans la notion selon laquelle la mort n'est pas voulue par Dieu, mais est entrée dans le monde par l'envie du diable (Sg 2,23-24), contrairement à la notion de la mort comme passage naturel dans la non-existence que les ennemis du héros de la Sagesse soutiennent en Sg 2,1-22. 
1) Puisque la résurrection corporelle est compatible avec l'eschatologie sapientielle, peut-être même implicite en elle, quoique non explicitement affirmée par elle, la résurrection corporelle pourrait facilement devenir explicite dans cette eschatologie si des circonstances spécifiques exerçaient sur le concept des pressions en ce sens, par exemple, l'expérience de Pâques des premiers disciples de Jésus.

2) Si la résurrection corporelle est effectivement devenue explicite (comme je crois qu'elle le devint) dans l'eschatologie sapientielle d'immortalité, elle n'aurait pas la même signification que dans l'eschatologie apocalyptique résurrectionnelle. On ne la verrait pas comme une légitimation de chaque persécuté, puisque cette justification a eu lieu dans la mort/exaltation même de l'Unique Juste. La résurrection n'apparaîtrait pas non plus comme une victoire sur la mort, parce que la mort n'a jamais eu de véritable pouvoir sur celui qui est un enfant de Dieu. La résurrection serait essentiellement une manifestation du sens, pour la personne corporelle, de la vie en Dieu maintenant vécue dans sa plénitude. Dans le cas de Jésus, comme nous verrons, ce serait une condition de possibilité de sa présence personnelle postpascale à ses disciples et de son action continue dans le monde.

Je suggère que la résurrection corporelle de Jésus en Jean est précisément présentée en termes d'eschatologie sapientielle. Le récit de résurrection en Jean 20 n'est donc pas une concession aux contraintes de la tradition chrétienne primitive. C'est une exploration narrato-théologique de l'expérience pascale faite par les premiers disciples et des implications de cette expérience pour la spiritualité de la communauté johannique et des disciples postérieurs. En d'autres mots, les deux dimensions du mystère pascal de Jésus, sa glorification sur la croix (c.-à-d. son passage de ce monde à son Père) et sa résurrection (c.-à-d. son retour promis aux siens), bien que reliées, ne sont pas strictement identiques en Jean. La glorification est la condition de possibilité de la résurrection. En conséquence, les apparitions en Jean ne concernent pas d'abord l'expérience postmortelle de Jésus, mais l'expérience que font ses disciples de son retour vers eux.

\subsection{L'anthropologie de Jean}

L'anthropologie du quatrième évangile est étroitement liée à son eschatologie. Beaucoup de discussions au sujet de la résurrection corporelle sont mal engagées, dès le départ, du fait que les Occidentaux modernes tendent à lire les textes de l'évangile sous l'angle d'une anthropologie philosophique fondamentalement dualiste et substantialiste. L'anthropologie 
de Jean, bien qu'exprimée avec le vocabulaire grec qui a clairement influencé sa compréhension de la personne, est complètement enracinée dans la langue et la sensibilité hébraïques ${ }^{15}$. Les termes grecs pertinents, $\psi v \chi \eta^{\prime}$

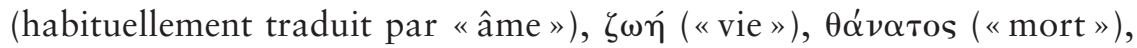
$\sigma \alpha \dot{\alpha} \rho \xi$ ("chair »), $\alpha \hat{i} \mu \alpha$ («sang»), $\pi \nu \in \hat{v} \mu \alpha$ (" esprit») et $\sigma \hat{\omega} \mu \alpha$ ("corps »), constituent un champ sémantique complexe dans lequel tous les termes sont interreliés et se qualifient mutuellement. Bien qu'en français, ces termes dénotent tous une composante ou un état de l'être humain, dans l'utilisation biblique, ils dénotent la personne entière dans une certaine perspective ou sous un certain aspect. Ignorer cette différence peut provoquer de sérieux malentendus, tels que la tendance de beaucoup de modernes à entendre des connotations cannibaliques dans l'invitation de Jésus à manger sa chair et à boire son sang en Jn 6,52-58. Il est crucial de comprendre ce que ces termes anthropologiques signifiaient dans le contexte du judaïsme du $\mathrm{I}^{\mathrm{er}}$ siècle, auquel se rattache Jean, afin de comprendre comment ils fonctionnent dans le quatrième évangile en général, mais particulièrement dans le récit de glorification et de résurrection de Jésus.

Les contraintes d'espace empêchent d'examiner chacun de ces termes, mais deux d'entre eux sont essentiels à notre propos: $\sigma \alpha \dot{\rho} \rho \xi$ en relation à $\alpha \hat{i} \mu \alpha$ («chair» et «sang ») et $\sigma \omega \hat{\omega} \mu \alpha$ («corps»). Pour des modernes, $\sigma \alpha \dot{\alpha} \rho \xi$ et $\alpha \hat{i} \mu \alpha$ dénotent deux composantes séparables de l'être humain, respectivement solide et liquide. Cependant, la chair, dans l'anthropologie de Jean, n'est pas une partie de l'humain distincte des os et du sang, mais la personne entière comme naturelle et mortelle ${ }^{16}$. Quand la chair est combinée avec le sang, elle renvoie à la personne mortelle par nature, mais réellement

15. Une bonne introduction à l'anthropologie sémitique est l'ouvrage de H.W. Wolff (1996). Comme nous avons vu en ce qui concerne son eschatologie, l'évangile de Jean n'est pas exempt d'influences hellénistiques, venant probablement de matériaux sapientiaux de l'Ancien Testament, en particulier la Sagesse de Salomon. Cependant, cette influence est contrôlée par des compréhensions hébraïques de Dieu, de l'humain et de la fin de la vie humaine. Une étude approfondie de l'anthropologie johannique, qui irait totalement au-delà de la portée de cet article, procéderait suivant un itinéraire qui irait de l'utilisation hébraïque concrète et stéréométrique (pour reprendre les termes de Wolff), en passant par les changements perçus dans les vocabulaires du grec de la LXX, jusqu'au quatrième évangile. Je suspecte que le développement le plus original soit précisément l'exploitation chez Jean de la distinction, impossible en hébreu mais possible en grec, entre $\sigma \alpha \dot{\alpha} \rho \xi$ et $\sigma \omega \hat{\omega} \mu \alpha$.

16. La «chair» est une bonne traduction de $\sigma \alpha \dot{\alpha} \rho \xi$, terme plus différencié que le bāśār hébraïque qui signifie l'humain dans son infirmité ou sa faiblesse (Wolff 1996, 26-31). Mais le terme hébreu couvre aussi la signification de «corps » presque complètement, tandis que le grec distingue $\sigma \alpha \alpha \rho \xi$ de $\sigma \omega \hat{\omega} \mu \alpha$, une distinction cruciale pour la théologie de la résurrection chez Jean. 


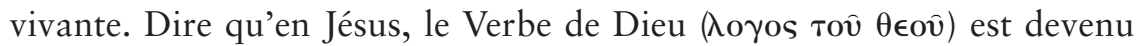
chair $(\sigma \alpha \dot{\alpha} \rho)$, c'est dire que le Verbe est devenu entièrement humain, c'està-dire mortel ${ }^{17}$. Dans les Psaumes particulièrement, nous voyons "chair " employé pour parler de l'humanité dans sa faiblesse et sa mortalité: «Dieu s'est rappelé qu'ils étaient chair, un souffle passager qui ne revient pas " (Ps 145,21 ; voir également 56,$5 ; 65,3 ; 145,21$; etc.). En Jn 6,51, Jésus dit qu'il est le pain vivant descendu du ciel, et que le pain qu'il donnera pour la vie du monde "est [sa] chair». Jésus ne parle pas d'une partie physique de luimême. Il dit qu'en se donnant lui-même totalement dans la mort — ce qui est seulement possible parce qu'il est chair, c'est-à-dire mortel —, il donne la vie au monde comme le pain donne la vie à celui qui le mange.

Le terme le plus important dans ce champ sémantique anthropologique, en relation à la résurrection de Jésus, est $\sigma \omega \hat{\omega} \mu \alpha$, "corps » : Jean l'emploie d'une manière subtile en mariant des compréhensions sémitiques et hellénistiques de l'humain ${ }^{18}$. Puisque les modernes ont tendance à penser le corps comme une substance distincte dans le composite humain, une composante physique qui se distingue du spirituel, ils tendent à le confondre avec la chair - elle-même mal comprise comme composante malléable mais solide, en contraste avec le sang et les os. En d'autres mots, le corps tend à être compris comme une substance physique, intégralement constitutive de la personne, mais n'en constituant qu'une partie ${ }^{19}$.

Pour Jean, le corps est la personne dans son autoprésentation symbolique. La personne peut être vivante ou morte ${ }^{20}$, mais c'est l'individu entier,

17. Pour un riche traitement du sens de "chair» en Jean, voir Lee 2002, 29-64.

18. Ici, je suis en désaccord avec Lee (2002, 45-46), qui suggère qu'il n'y a aucune différence significative entre $\sigma \alpha \dot{\rho} \rho \xi$ et $\sigma \bar{\omega} \mu \alpha$. Mon argument est qu'il y a une différence essentielle: Jésus ne se lève pas en tant que "chair» $(\sigma \alpha \dot{\alpha} \rho \xi)$, mais en tant que «corps» $(\sigma \omega \hat{\omega} \mu \alpha)$.

19. Il est intéressant que la médecine psychosomatique découvre de diverses manières comment l'humain entier est complètement "corps ", non dans le sens réducteur de n'être rien sauf de la matière physique, mais dans le sens d'être, globalement, une personne corporelle. Cette compréhension est plus près de la compréhension biblique que l'anthropologie réductionniste engendrée par la révolution scientifique et le Siècle des lumières. Néanmoins, les compréhensions contemporaines de l'humain demeurent très dichotomiques, comme il est démontré par les approches souvent mécanistes des procédures médicales.

20. L'influence hellénistique sur la pensée de Jean aussi bien que l'exploitation des possibilités de la langue grecque sont claires ici. Bāśār n'est pas employé pour parler d'un cadavre (bien que le nepeš soit utilisé de temps en temps en ce sens), mais seulement pour parler de créatures vivantes, tandis que Jean n'emploie pas $\sigma \alpha \dot{\alpha} \rho \xi$ (que la LXX utilise pour traduire $b \bar{a} \bar{s} \bar{a} r$ ), mais $\sigma \widehat{\omega} \mu \alpha$ pour parler des cadavres sur la croix (Jn 19,31) et spécifiquement du corps mort de Jésus (Jn 19,38.40;20,12) ainsi que de son corps ressuscité $(2,21-22)$. 
le «soi corporel », qui est vivant ou mort. Dans la pensée sémitique, une fois que le corps de la personne morte commence à se décomposer et à s'effriter, la personne n'est plus une personne. Quelle que soit la trace de l'individu qui peut survivre au shéol, il ne s'agit pas d'un être humain, parce qu'il ne jouit pas de la subjectivité, de la communauté ou de l'union avec Dieu $^{21}$. Le corps est la quintessence de la personne en tant que symbolisation de soi, c'est-à-dire en tant que numériquement distincte, cohérente et permanente: un sujet qui peut interagir avec d'autres sujets et qui est présent et actif dans le monde ${ }^{22}$. Un cadavre, dans le vocabulaire de Jean, s'appelle aussi un corps (Jn 19,31.38.40; 20,12), précisément parce qu'il symbolise la personne entière, le "soi corporel ", dans sa transition de l'être au non-être ou de la présence à l'absence. Le cadavre est la personne symbolique, perceptiblement réelle, en train de devenir absente. Quand la personne est finalement entièrement absente, quand le cadavre s'est décomposé - ce qui, la chose est importante, ne se produit pas dans le cas de Jésus -, il n'est plus considéré comme un corps. En bref, si le Jésus prépascal comme chair, c'est-à-dire en tant qu'être humain mortel, était la présence symbolique de la gloire de Dieu en ce monde (voir Jn 1,14), Jésus comme corps est sa propre présence symbolique à ses contemporains. Avant sa mort, les deux aspects, charnel et corporel, représentent la personne humaine mortelle appelée Jésus, et sont concomitants, comme ils le sont dans tous les humains en cette vie. La question du «corps» comme distinct de la «chair» surgit seulement quand Jésus meurt et que les deux ne sont plus concomitants ${ }^{23}$.

21. Je n'ai jamais vu une meilleure définition du shéol que celle de J.L. McKenzie, pour qui le shéol «est moins un concept positif de survie qu'une négation pittoresque de tout ce qui est signifié par «vie» et «activité»» $(1965,800)$.

22. J'ai longuement traité, par ailleurs, du concept de symbole, particulièrement de son fonctionnement en Jean (2003, 63-77; voir aussi Lee 2002, 9-28). Karl Rahner (1966, spécial. 245-252) a fait un travail très important sur le symbole en théologie et particulièrement sur le corps comme symbole premier par lequel une personne est présente à lui-même/elle-même comme à d'autres. J.A. Bracken (2004) dialogue avec la position de Rahner du point de vue de la métaphysique néowhiteheadienne. Il est particulièrement intéressant que le terme «corps " ne semble pas jouer un rôle vraiment distinct dans la pensée sémitique pour mériter un terme propre qui le distinguerait de la «chair ». Les seuls corps connus à l'expérience humaine étaient les corps charnels, que ce soit l'humain potentiel, la «créature terrestre ( hāādām) de Gn 2,7 ou la personne vivante, nepeš ou bāśār.

23. Je trouve très suggestive la remarque faite par M. Coloe $(1999,4, \mathrm{n}$. 6): «En parlant de Jésus à la fois comme Temple et Tabernacle il n'y a pas de dichotomie, car tous deux sont intrinsèquement liés, comme la chair $(1,14)$ est liée au corps $(2,21)$. Le Tabernacle et le Temple remplissent la même fonction symbolique même s'ils rappellent différentes 
La question de la présence réelle de Jésus dans et après son passage par la mort domine le dernier repas en Jean (chap. 13 à 17), aussi bien que le récit de résurrection (chap. 20). Où est le Seigneur ? Est-il allé là où ses disciples ne peuvent pas le suivre? Sont-ils des orphelins, privés de la gloire de Dieu qui avait été présente dans la chair de Jésus? Les futurs croyants sont-ils condamnés à une foi fondée sur une rumeur au sujet d'événements auxquels ils n'ont pas participé et ne participent pas? À moins que Jésus soit ressuscité corporellement, à moins qu'il soit vivant dans la pleine intégrité de son humanité, donc, à moins qu'il ne soit symbolisé corporellement, il n'est pas présent, que ce soit comme présence de l'humanité en Dieu ou comme présence divinement humaine de Dieu en nous.

L'enjeu anthropo-théologique crucial en ce qui a trait à la résurrection est la relation de la chair au corps, la relation de la personne prépascale de Jésus comme être humain mortel à la personne postpascale de Jésus comme Fils de l'Homme glorifié. En anticipant et de manière résumée, je propose que la relation de la chair au corps est précisément ce qui est changé par la glorification de Jésus. Dans son existence prépascale comme chair, le corps de Jésus (sa présence symbolique personnelle) a été conditionné par sa mortalité. Il était sujet à la mort et aux limitations de l'espace, du temps et de la causalité que la vie humaine naturelle entraîne. Dans sa glorification, Jésus va au Père comme un "soi corporel » humain et, dans sa résurrection, il revient vers les siens dans la pleine intégrité de son humanité. Son corps est réel, à la fois en continuité et en discontinuité avec son corps prépascal. Dire que Jésus glorifié et ressuscité est une personne corporelle, c'est affirmer qu'il est numériquement distinct, un sujet personnel qui peut être présent et actif dans un échange intersubjectif ${ }^{24}$, mais qu'il n'est plus chair. Et il sera présent dans ce même corps tout au long du temps postpascal par la gamme des symboles à travers lesquels sa présence personnelle sera manifestée.

périodes historiques. » En fait, je pense que la chair et le corps dénotent des modes différents et subséquents (analogues aux périodes historiques) de la présence de Jésus à ses disciples. La chair indique sa vie comme un mortel et le corps, sa vie glorifiée. Mais les deux termes dénotent la même personne et la même présence de la gloire de Dieu parmi les humains, en cette personne.

24. Ward (1999, spécial. 168; voir aussi 1998), dans un article fascinant qui étudie le corps de Jésus dans ses déplacements, transformations et changements de signification, jette un éclairage postmoderne qui confirme notre analyse de ce thème. 


\section{La structure et les dynamiques du récit de résurrection johannique}

De plus en plus, les chercheurs johanniques reconnaissent que le récit de la résurrection du quatrième évangile n'est pas une collection aléatoire d'épisodes interchangeables, mais une unité littéraire/théologique organique et que les indices de signification se situent dans sa structure ${ }^{25}$ (Lee 1995). C'est là cependant que le consensus prend fin. Les chercheurs ont proposé un nombre surprenant de structures très plausibles pour Jean 20, ce qui inclut des structures chronologiques et géographiques, numérologiques et verbales, narratives et dramatiques, théologiques et spirituelles, dont beaucoup sont complémentaires plutôt que contradictoires, même si elles mènent à différentes interprétations ${ }^{26}$. Évidemment, nous ne pouvons pas toutes les passer en revue ici, mais je proposerai deux structures complémentaires, une théologique et une spirituelle, sans supposer l'accord ou le désaccord avec d'autres théories. Ma proposition est au service du propos de cet article particulier, à savoir répondre à la question : quelle fonction le corps de Jésus glorifié joue-t-il dans la relation entre Jésus et ses disciples postpascals?

\subsection{La structure théologique de Jean 20}

Le récit débute aux versets 1 et 2 . Marie de Magdala vient au tombeau dans l'obscurité (toujours symboliquement négative chez Jean), aperçoit que la pierre a été enlevée du tombeau et rapporte à Simon Pierre et au disciple bien-aimé qu' »ils ont enlevé le Seigneur du tombeau et nous ne savons pas où ils l'ont mis» (20,2). Le "nous ", bien que Marie était seule au tombeau, souligne que le problème n'est pas seulement personnel, mais

25. Bien que Lee $(1995,38-40)$ considère la structure comme une indication importante de signification, elle ne regarde pas la première scène, celle du disciple bien-aimé et de Simon Pierre au tombeau, comme vraiment intégrée à la signification du récit de résurrection johannique. Elle considère cette péricope comme une prolepse mineure préparant le chap. 21. Je suis en désaccord avec cette position, bien que je trouve éclairant son traitement de Marie de Magdala et de Thomas en tant qu' »associés narratifs » dans la foi de Pâques entourant l'épisode central de l'apparition aux disciples.

26. Récemment, R. Crotty (1999) a résumé et critiqué un certain nombre d'essais majeurs pour déchiffrer la structure de Jn 20 : Moloney (1998, 516); Lee (1995); Byrne (1985); La Potterie (1984); Mollat (1974); Dupont et coll. (1973); Brown (1970, 965). Cette liste est loin d'être exhaustive. Crotty, bien sûr, offre sa propre structure du chapitre. 
communautaire ${ }^{27}$. La question programmatique qui donne son impulsion à la première moitié du récit de résurrection johannique (v. 3-18) est énoncée: "Où est le Seigneur ", après sa mort ? La question suggère aussi une réponse possible: Jésus est un cadavre; il est vraiment parti.

Dans l'épisode suivant (v. 3-10), Simon Pierre et le disciple bien-aimé courent au tombeau. L'évangéliste structure soigneusement cette histoire pour que Pierre entre d'abord dans le tombeau et voie son contenu: les linges mortuaires et le suaire ( $\sigma o v \delta \alpha ́ \alpha \rho o v)$ de Jésus, qui ne se trouve pas avec les linges, mais est soigneusement roulé et clairement placé de côtée ${ }^{28}(20,7)$. Le disciple bien-aimé entre en deuxième et voit ce qu'il n'a pas vu de l'extérieur quand il a regardé la première fois. De l'extérieur, il n'avait vu que les linges $(20,5)$. À l'intérieur, il voit aussi le suaire et on nous dit qu'il «vit et il crut ", une expression que Jean emploie pour signaler la réponse de foi appropriée à un signe (par ex. : 2,23; 6,30;11,40).

Ce signe, le suaire maintenant étendu de côté, est à la fois en continuité et en discontinuité avec les signes faits par Jésus dans son ministère public. Une caractéristique des signes dans les deux régimes est qu'ils sont symboliques et donc intrinsèquement ambigus. Tous ceux présents ont vu l'aveuglené guéri au chapitre 9 et Lazare redressé au chapitre 11. Certains ont vu et ont cru. D'autres ont vu et n'ont pas cru. Dans cet épisode, Simon Pierre et le disciple aimé voient le suaire. Le disciple bien-aimé croit, mais pas Pierre.

Or, ce signe se distingue aussi de ceux exécutés par le Jésus prépascal. Jésus lui-même n'est pas visiblement présent réalisant une œuvre. Dans cet épisode, les disciples se voient offrir comme signe un objet qui doit être interprété comme révélateur, probablement dans la ligne du voile de Moïse, que celui-ci a porté pour protéger les Israélites de la gloire de son visage, mais qu'il enlevait quand il était «face à face » avec $\operatorname{Dieu}^{29}$ (Ex 34,29-35).

27. Crotty $(1999,159)$ suit plusieurs commentateurs qui considèrent le «nous " comme une "aporie ", une trace d'un texte source qui, à l'origine, incluait d'autres femmes ayant accompagné Marie de Magdala au tombeau (comme on le constate dans chacun des trois récits synoptiques de résurrection). Cependant, à la différence de plusieurs, Crotty propose que Jean utilise le «nous » pour faire de Marie de Magdala une représentante de la communauté, par opposition aux «ils » qui ont pris le Seigneur (164). Je préfère cette position à l'attribution d'une maladresse rédactionnelle à un auteur de la compétence de Jean. Je pense également que cette interprétation est porteuse d'une charge ecclésiologique.

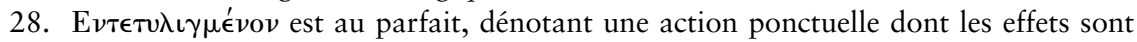
permanents ou qui dure dans ses effets.

29. Pour l'argumentation linguistique concernant l'arrière-plan vétérotestamentaire de

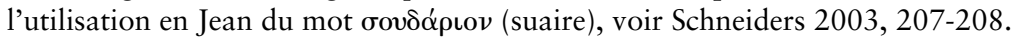


La finale de la scène (v. 9), cependant, a souvent défié les exégètes: «En effet, ils ne savaient pas encore que, d'après l'Écriture, il devait ressusciter d'entre les morts. » Ainsi, si ce n'est pas en la résurrection, en quoi le disciple bien-aimé a-t-il cru ? Je suggère qu'il a cru à ce que Jésus avait dit plusieurs fois à propos de sa mort (par ex. : 13,1; 16,28; 17,1; 17,24), à savoir que c'est par cela qu'il serait glorifié. Le disciple bien-aimé a cru que sur la croix, bien qu'il soit vraiment mort, Jésus a été exalté en présence de Dieu. Le suaire de sa chair, c'est-à-dire la mortalité dans laquelle sa gloire avait été voilée pendant sa vie prépascale, est maintenant irrévocablement mis de côté. Jésus, nouveau Moïse, est monté sur la montagne pour sceller la Nouvelle Alliance entre Dieu et le Nouvel Israël.

Le lecteur a maintenant le début de la réponse à la question: "Où est le Seigneur ? » Il est avec Dieu. Il est glorifié. Mais il y a plus, quelque chose que les disciples ne comprennent pas encore, à savoir que Jésus n'est pas seulement glorifié, mais ressuscité d'entre les morts.

Dans l'épisode suivant (v. 11-18), Marie de Magdala est encore au tombeau. Cette scène est imprégnée d'allusions au jardin de la première création et particulièrement au lieu de rendez-vous mentionné par le Cantique des cantiques, la chanson nuptiale de l'alliance entre YHWH et Israël. Dans le tombeau, Marie ne voit pas les linges et le suaire, mais deux anges, un à la tête et un aux pieds de l'endroit où le corps $(\mathrm{sw}=\mathrm{ma})$ de Jésus avait été placé. Cette description, et même les mots employés, rappellent le trône d'or, le "siège de miséricorde " de l'arche de l'Alliance (voir Ex 37,6-9 TM = Ex 38,5-8 LXX) qui était gardé par deux chérubins, l'un et l'autre au bout «du lieu de rencontre de Dieu et des humains" (Clifford 1990, 56). Marie de Magdala pleure avec désolation, en l'absence de Jésus qu'elle assimile clairement à un cadavre qui a été emporté. Quand Jésus, le bon Berger, l'appelle par son nom, elle se tourne, elle est convertie, elle passe du désespoir à la reconnaissance de Jésus comme le "maître » qu'elle avait connu dans sa vie prépascale. Il y a infiniment plus dans cette scène fort riche, mais pour l'objectif qui est le nôtre, ces observations fournissent le deuxième volet de la réponse à la question: "Où est le Seigneur? " Il est revenu vers les siens. Quand Marie arrive en proclamant ( $\left.\alpha \gamma \gamma \in \epsilon^{\prime} \lambda \lambda o v \sigma \alpha\right)$ l'Évangile de Pâques à ceux qui sont maintenant les «frères et sœurs » de Jésus, elle dit explicitement: "j'ai vu le Seigneur» $(20,18)$. Par un signe, le disciple aimé a vu et a cru que Jésus a été glorifié, vivant avec Dieu. Marie de Magdala a fait l'expérience de Jésus ressuscité, rendu aux siens.

Avec la proclamation de l'Évangile de Pâques, selon lequel Jésus est glorifié et ressuscité, le récit entre dans sa deuxième phase (v. 19-29), dont 
le cadre n'est pas l'aube de l'ère nouvelle dans le jardin du tombeau et les protagonistes ne sont pas les premiers apôtres, mais plutôt le soir du premier jour de cette ère nouvelle, à Jérusalem « où les disciples étaient réunis » comme communauté. La question «Où est le Seigneur? » laisse place maintenant à la question qui domine la deuxième moitié du récit de résurrection johannique: "Comment peut-on faire l'expérience du Seigneur ressuscité ?" La première scène de cette deuxième partie (v. 19-23), la venue de Jésus à la communauté, est la pièce maîtresse du récit de résurrection johannique. En dépit des portes verrouillées, Jésus se dresse au beau milieu de la com-

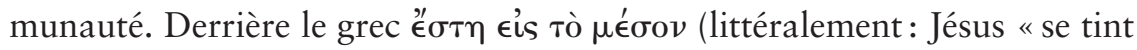
au milieu " de la communauté) se profile le verbe araméen pour "se lever ", qui peut faire allusion au fait de se dresser physiquement ou au fait de ressusciter d'entre les morts. Comme il avait promis dans son premier acte public dans le Temple de Jérusalem, Jésus, le troisième jour, élève le nouveau temple de son corps au milieu de la communauté. Dans le Temple, les adversaires de Jésus l'avaient mis au défi: "Quel signe nous montres-tu $[\ldots]$ ? Jésus leur répondit: «Détruisez ce temple et en trois jours je le relèverai.» [...] Mais lui parlait du temple de son corps $\sigma \omega \hat{\omega} \mu \alpha$. Aussi, quand il ressuscita d'entre les morts, ses disciples se rappelèrent qu'il avait dit cela, et ils crurent à l'Écriture et à la parole qu'il avait dite. » (Jn 2,18-22)

La scène se déploie alors en deux actions, toutes deux inaugurées par le «Paix à vous » de Jésus, réalisant sa promesse de leur donner, à son retour, une paix que le monde ne peut pas donner (voir 14,27;16,33). Le

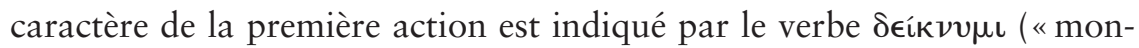
trer» ou «manifester»), terme johannique dénotant la révélation. Jésus leur montre ses mains et son côté, c'est-à-dire leur révèle le sens pour eux de sa glorification et ils se réjouissent de cette révélation que le Seigneur luimême est effectivement au milieu d'eux, glorifié mais toujours marqué par les signes de son mystère pascal. Son «soi corporel » est à la fois en continuité et en discontinuité avec celui qu'ils avaient connu, Jésus, qui avait promis que son départ constituerait une nouvelle venue vers eux ${ }^{30}(14,28)$.

30. Les deux verbes de ce texte sont au présent. On pourrait s'attendre à : "après que je sois parti, je reviendrai à toi », mais à la place nous avons littéralement: «je pars et je viens à toi ». Ce qui pourrait être paraphrasé comme suit: "mon départ est ma venue vers toi ", c'est-à-dire ma séparation de toi dans la chair est ma venue vers toi dans l'Esprit. En fait, voilà pourquoi il est avantageux ou nécessaire pour les disciples que Jésus parte (voir Jn 16,7). Dans la chair, il ne pourrait pas établir le genre d'intériorité mutuelle avec ses disciples qu'il peut établir dans l'Esprit qui sera avec eux et en eux (voir Jn 14,16-17). 
La deuxième action, suivant le don répété de la paix, est un mandat confié à ce nouveau Peuple, de même que Dieu avait mandaté Jésus. Il souffle sur eux et dit: «Recevez l'Esprit saint ». Le verbe «souffler» ('́ $\mu \phi v \sigma \alpha \dot{\alpha} \omega)$ est un hapax legomenon présent seulement ici dans tout le Nouveau Testament. On ne le retrouve que deux fois ${ }^{31}$ dans l'Ancien Testament: en Gn 2,7, quand Dieu, lors de la première création, insuffle la vie à la créature terrestre qui devient le premier être humain vivant, et en Ez 37,9-10, quand le prophète au nom de Dieu insuffle la vie aux ossements desséchés pour recréer, relever des morts, le peuple d'Israël. Dans cette scène de Pâques, ce "souffle» apparaît pour la troisième fois dans la Bible, lorsque Jésus insuffle l'Esprit promis de la Nouvelle Alliance dans la communauté des disciples, les créant ainsi en tant que Nouvel Israël.

La structure de cette scène est celle de l'expérience de l'Alliance au Sinaï, dans laquelle la grande théophanie sur la montagne a été suivie du don de la Loi qui a fait d'Israël le Peuple de Dieu. Par le prophète Ézéchiel, Dieu avait promis une Nouvelle Alliance:

Je conclurai avec eux une alliance de paix, ce sera avec eux une alliance éternelle. Je les établirai, je les multiplierai et j'établirai mon sanctuaire au milieu d'eux à jamais. Je ferai ma demeure au-dessus d'eux, je serai leur Dieu et ils seront mon peuple. Et les nations sauront que je suis Yahvé qui sanctifie Israël, lorsque mon sanctuaire sera au milieu d'eux à jamais. (Ez 37,26-28; voir 34,25 ; Is 54,10$)$.

Dieu apparaît maintenant non dans le tonnerre et les éclairs, mais dans la personne de Jésus glorifié et ressuscité. Et comme promis en Ez 36,27-28 — « Je mettrai mon esprit en vous et je ferai que vous marchiez selon mes lois» - l'Esprit saint, la nouvelle Loi, est versé dans leurs cœurs. Jésus, comme Dieu avait promis, établit ici la Nouvelle Alliance avec le Nouvel Israël, en élevant au milieu de ce Nouvel Israël le Nouveau Temple de son corps. Ainsi est fondée l'Église, mandatée pour continuer la mission de Jésus: faire disparaître le péché du monde et garder tous ceux que Dieu lui avait donnés. Il est important de noter que l'évangéliste ne nous dit pas que Jésus, ayant accompli son œuvre, part ou s'en va. Jésus est irrévocablement revenu vers les siens. Il viendra et reviendra de nouveau, mais sans

31. En fait, le verbe apparaît quatre fois dans la LXX: les deux exemples apportés ici, ainsi que Sg 15,11 qui rappelle le souffle vital d'Adam et $1 \mathrm{R}$ 17,21 qui raconte la réanimation du fils de la veuve de Sarepta par le prophète Élie. La LXX, de façon inexacte (mais peut-être délibérément) traduit le mot hébreu correspondant à "étiré » ou «mesuré » par le grec « respiré », peut-être pour faire référence au récit de création. 
jamais quitter. Qu'il soit présent et qu'il sache ce qui survient dans sa communauté est clair dans ce qui suit.

À première vue, la scène finale du récit de la résurrection johannique (v. 24-29), l'épisode de Thomas - qui se prolonge aux v. 30-31 avec la conclusion de l'évangile - semble inappropriée ${ }^{32}$. Rien dans la scène précédente n'a suggéré que quelqu'un était absent quand Jésus est apparu dans la nuit de Pâques. Thomas est identifié comme «le jumeau ${ }^{33}{ }^{3}$. Sa double identité est immédiatement spécifiée : positivement, il est l' "un des Douze », compagnon du Jésus prépascal et participant aux signes prépascals; négativement, il n'était "pas avec" les disciples réunis la nuit de Pâques où Jésus est apparu - il est donc un de ceux qui connaîtront la résurrection non par une expérience pascale, mais par le témoignage de l’Église: «nous avons vu le Seigneur ».

Thomas refuse cette nouvelle structure de foi, il refuse d'entrer dans ce nouveau régime. Il insiste sur le fait qu'il croira seulement s'il peut toucher les blessures mêmes de Jésus, s'il peut revenir au régime de foi prépascale, s'il peut continuer à se rapporter au Jésus dans la chair. Notons que Thomas ne confond pas spontanément le Jésus glorifié avec le Jésus prépascal comme Marie de Magdala l'avait fait. Il demande que Jésus soit pour lui comme il avait été avant la glorification. Et notons encore que Thomas «ne doute pas» comme cela est si souvent affirmé. Il refuse: «je ne croirai pas ». Dans l'évangile de Jean, croire et refuser de croire sont toujours une question de libre choix et non la réponse naturelle à l'évidence irréfutable ou à son manque.

Jésus vient de nouveau, une semaine plus tard, le dimanche soir, au temps des célébrations eucharistiques de l'Église primitive. De nouveau, il

32. Bien que je ne puisse pas traiter de cela ici, il y a une hypothèse qui paraît avoir un certain mérite, à l'effet que cet épisode pourrait avoir eu des visées antignostiques. Marie de Magdala et Thomas sont des figures importantes dans la littérature gnostique et ils jouent des rôles très significatifs dans l'évangile de Jean, qu'ils ne jouent pas dans les récits synoptiques de résurrection. Voir A.D. DeConick (1997) qui propose que l'épisode de Thomas a été composé pour réfuter les chrétiens thomasiens en Syrie dont la sotériologie caractéristique apparaît dans l'évangile de Thomas. Elle propose que l'évangile de Thomas puisse dater de 70-80 apr. J.-C. et pourrait ainsi avoir été à la disposition du quatrième évangéliste.

33. M. de Jonge $(1978,119)$ appelle Thomas « un cas limite », parce qu'il est le dernier de ceux qui ont vu des signes et le premier de ceux qui doivent croire à partir de la parole de témoins. Je conviens qu'il se tient sur la frontière entre l'expérience de Pâques et l'expérience postérieure du Jésus ressuscité, mais je ne pense pas que les signes sont remplacés par la parole des témoins. C'est plutôt un genre de signe qui laisse la place à un nouveau genre de signe. 
"se lève au milieu d'eux », leur souhaite la paix, mais cette fois, bien que les portes soient fermées, marquant ainsi les limites de la communauté ecclésiale, il n'y a aucune mention de peur des autorités. Le don initial de la paix par Jésus a éliminé la peur. Nous sommes dans le temps postpascal. Mais Jésus s'adresse directement à Thomas dont il sait parfaitement les pensées intérieures et les mots extérieurs. "Je connais les miens et les miens me connaissent » (Jn 10,14).

Il invite Thomas non à faire ce que Thomas avait demandé — sonder physiquement les blessures dans ses mains et son côté pour vérifier sa résurrection physique -, mais à faire l'expérience, différente mais tout aussi juste et réelle, de son identité véritable. Il dit: "Mets ici ton doigt et vois mes mains». Or, on ne "voit» pas avec son doigt. L'impératif i1de ( «contemple» ou «voit»), comme M. de Goedt (1962, 142-150) l'a montré il y a plusieurs années, fonctionne dans le quatrième évangile comme élément d'une formule de révélation. L'invitation n'est pas de voir physiquement, mais de saisir ce qui ne peut pas être vu avec les yeux de chair (par exemple, que Nathaniel soit un vrai Israélite sans ruse, ou que le disciple bien-aimé au calvaire soit maintenant le vrai fils de la mère de Jésus). Les blessures de Jésus ne sont pas une preuve de réalité physique, mais la source d'une vraie compréhension du sens de la mort révélatrice de Jésus.

Alors, l'invitation prend une portée plus profonde. Jésus commande à Thomas de mettre sa main dans son côté ouvert, duquel étaient sortis l'eau et le sang vivifiants, symbole du don de l'Esprit au baptême et de l'eucharistie que Jésus avait transmise dans sa mort et qu'il avait condensée dans un don à la communauté, une semaine plus tôt, lorsque Thomas était

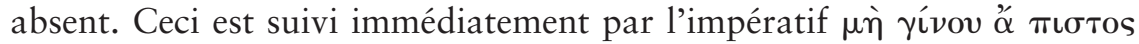

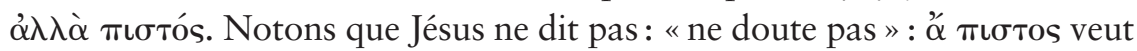
dire "refuser de croire », "être infidèle », "être traître ». La réponse immédiate de Thomas - non une tentative de toucher Jésus physiquement, mais une reconnaissance de ce qu'il peut saisir seulement par la foi - explique sa conversion du statut d'infidèle au statut de fidèle, son passage d'un attachement entêté à la chair de Jésus ( «à moins que je ne le touche physiquement, je ne croirai pas!»), au don de lui-même qu'il fait au Ressuscité («Mon Seigneur et mon Dieu!»). En d'autres termes, Jésus dit à Thomas non ce que le Jésus de Luc dit à ses disciples qui n'en croient leurs yeux tant leur joie les surprend: "palpez-moi et voyez que je suis chair et os, que je ne suis pas un fantôme", mais plutôt: "Thomas, saisis dans la foi ce que ma mort salvatrice signifie et approprie-toi dans la foi les fruits de cette 
mort, l'Esprit versé de mon côté ouvert ». Il dit en fait ce qu'il a dit à Simon Pierre au dernier repas: "à moins que tu n'entres par la foi dans le nouveau régime inauguré par ma glorification, tu ne peux pas avoir part avec moi. »

Jésus accueille la conversion de Thomas sans réserve et confirme sa foi de Pâques par une des deux seules béatitudes du quatrième évangile. Jésus rend équivalents les deux genres de croyance. La foi fondée sur la vue des signes prépascals, qui était appropriée dans le premier régime, est supplantée par la foi postpascale, qui sera fondée sur une nouvelle sorte de signes, comme le suaire plié, le témoignage apostolique de Marie de Magdala et du reste des disciples, les mots de l'Écriture — signes qui seront maintenant accomplis par l'intermédiaire de l'Église. "Bénis soient ceux [en incluant maintenant Thomas] qui n'ont pas vu et qui ont cru. » Ce ne sont pas les disciples qui viendront plus tard qui sont assimilés à Thomas, mais Thomas qui est assimilé à eux. L'ère prépascale est finie, même pour ceux qui y ont participé.

L'évangéliste conclut alors l'évangile en s'adressant directement aux disciples du régime postpascal. Le Jésus prépascal, dit l'évangéliste, a fait beaucoup de signes visibles, dont seulement quelques-uns sont écrits dans l'évangile. Mais l'évangile écrit a exactement la même fonction pour la foi des disciples venant plus tard que les signes que Jésus a exécutés en Palestine pour la génération apostolique ${ }^{34}$. En croyant, ces disciples venant plus tard auront la vie dans le nom de Jésus tout comme l'ont eue ses compagnons prépascals. Contrairement à ce que certains exégètes voudraient suggérer, en insérant une conjonction adversative entre les deux parties du v. $29-$ à savoir que Jésus dénigre la foi de Thomas basée sur le voir et exalte la foi de ceux qui n'ont pas vu de signes et qui croient - Jésus n'alloue de supériorité ni à l'expérience de la première génération ni à celle des disciples venant après. Comme Dorothy Lee $(1195,48)$ a bien dit, la foi des futurs croyants est dépendante du témoignage de la communauté apostolique, mais n'est d'aucune façon limitée par cette dépendance: "La confession de Thomas est un pont narratif entre le dimanche de Pâques et la vie de la communauté croyante". La pointe n'est ni que cette foi en réponse aux signes est défectible ni que cette expérience sensorielle (voir, entendre et toucher) n'aura plus de rôle dans la foi. Le mode du voir, et non le fait de

34. P.J. Judge (1992), s'appuyant sur U. Schnelle et D.A. Carson, soutient que l'incident de Thomas lui-même est un signe et que l'évangile fonctionnera de la même manière pour les croyants subséquents. 
voir, doit changer, parce que le mode de la présence physique de Jésus à ses disciples a changé, mais non le fait de sa présence physique.

\subsection{La dialectique de l'expérience sensorielle et de la croyance}

Nous nous tournons maintenant, très brièvement et pour la rendre explicite, vers la dialectique entre l'expérience sensorielle et la croyance, dialectique qui émerge comme la structure spirituelle du récit de résurrection johannique - avant de tirer des conclusions sur le corps de Jésus ressuscité, spécialement dans les incidents de Marie de Magdala et de Thomas.

Après les versets introductifs dans lesquels la question «Où est le Seigneur? " a été présentée, nous avons une scène dans laquelle Jésus n'apparaît pas visiblement. Le disciple bien-aimé vient à la foi dans la glorification de Jésus en rencontrant un signe, le suaire plié. À l'autre extrémité du récit de résurrection johannique se trouve la conclusion de l'évangéliste assurant aux disciples venant plus tard que, dans l'Écriture — dans laquelle non plus Jésus n'apparaît pas visiblement - , Jésus est vraiment et salvifiquement rencontré. En d'autres mots, au commencement et à la fin du récit de résurrection, il y a une rencontre avec Jésus à travers les signes, réalités matérielles sensibles dans lesquelles Jésus n'apparaît pas sous sa forme visible. Ces deux scènes racontent des expériences qui sont historiquement réalistes, le genre d'expérience que les croyants ont en " temps ordinaire ", lorsqu'ils goûtent le pain et le vin, entendent des mots, touchent l'eau.

Se déplaçant vers le cœur du récit, nous trouvons trois scènes qui surviennent dans le «temps extraordinaire » et qui sont clairement une narrativisation théologique de l'expérience spirituelle, réelle mais non physique, plutôt que le compte-rendu d'événements humains ordinaires survenant dans un temps ordinaire. En plein milieu, il y a la scène de l'établissement de la communauté d'alliance qui sera dans le monde le mode ordinaire de la présence et de l'action du Jésus glorifié, c'est-à-dire qui sera son corps. Assurée de l'identité de Jésus et de sa présence, et animée par son Esprit, la communauté pardonnera les péchés et tiendra dans la communion tous ceux que Dieu lui confiera, de la même manière que Jésus a fait disparaître le péché du monde (voir 1,29 ) et a gardé tous ceux que le Père lui avait donnés (voir 6,$37 ; 6,39 ; 10,27-29 ; 17,12 ; 18,9$ ).

Deux épisodes encadrent ce récit historicisé de la fondation de l'Église comme présence pleinement réalisée, corporelle quoique non physique, et définitive de Jésus au monde. Ces deux épisodes se produisent dans un espace/temps "entre deux", que nous pourrions appeler le temps de 
Pâques, quand Jésus est à la fois présent et absent. Le but de ces épisodes est de dévoiler narrativement la relation intrinsèque entre Jésus lui-même, comme personne corporelle distincte, et la communauté ecclésiale, qui est son corps dans le monde. Autrement dit, ces épisodes concernent le rapport de Jésus au Christ, médiatisé par la catégorie du " corps » : le corps de Jésus ressuscité qui est le principe du "corps du Christ» ecclésial. Les deux sont identiques, bien que non réductibles; distincts, mais inséparables. Il est temps, donc, d'examiner ces deux scènes en regard des commandements apparemment contradictoires que Jésus donne à Marie de Magdala, de ne pas le toucher, et à Thomas le Jumeau, de le toucher.

\section{Conclusions : toucher Jésus ressuscité ?}

Autant Marie de Magdala que Thomas le Jumeau subissent une conversion qui consiste à se détourner d'un mode d'expérience qui n'est plus possible et à se tourner vers un mode nouveau, non familier mais tout aussi réel, pour faire l'expérience de Jésus. Tous les deux, en réponse à un impératif négatif de Jésus (ce qu'ils ne doivent pas faire: ne me touche pas, ne sois pas incrédule) suivi d'un impératif positif (ce à quoi ils sont maintenant appelés : trouve-moi dans la communauté, reconnais-moi en croyant), doivent passer du régime prépascal au régime postpascal. Mais l'accent dans chaque épisode est différent. Les deux acteurs ont des positions différentes dans l'histoire: Marie de Magdala relie le temps prépascal et le temps de Pâques, Thomas le Jumeau relie Pâques et le temps postpascal. Leurs expériences répondent aux deux questions à l'ordre du jour : où rencontre-t-on le Seigneur? et comment rencontre-t-on le Seigneur?

Marie de Magdala est la première disciple prépascale à rencontrer le Seigneur ressuscité. Elle croit à tort que le régime passé a été réinstallé. Les choses seront comme elles avaient toujours été. Le malentendu littéral dans l'évangile de Jean est une technique littéraire pour décrire la croissance dans la foi. Marie tente de toucher Jésus, de se lier à lui comme elle le faisait dans le passé, en utilisant une formule de politesse convenant à cette époque: «Rabbouni », mais Jésus empêche sa tentative: «Ne me touche pas».

Il n'y a aucune base textuelle en faveur de l'opinion souvent exprimée selon laquelle Marie s'accrocherait de façon hystérique à Jésus ou essaierait de le retenir dans sa montée ${ }^{35}$. Le verbe est $\alpha$ $\alpha \tau \omega$, «toucher», et non

35. Voir Lee $(1995,42)$, qui est contre cette interprétation, cependant défendue par F.J. Matera $(1989,405)$ et T. Okure $(1992,180)$. 


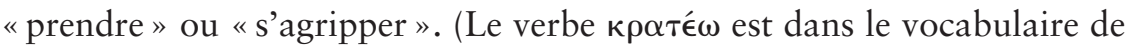
Jean et il l'utilise dans son sens normal dans la scène suivante, en 20,23.) La réponse de Jésus est $\mu \eta \mu$ ov $\alpha$ $\alpha \pi \tau o v$. Le verbe impératif est au présent, reflétant une activité en cours ou une activité continue, et la particule négative est en position emphatique : cette activité habituelle doit cesser. Le message est que le «toucher" physique - une métonymie qui convient à l'expérience de rencontre de deux personnes, expérience historique médiatisée physiquement "dans la chair ", c'est-à-dire à titre d'êtres humains mortels - est terminé. Jésus dit: "Va vers mes frères et sœurs". L'endroit où Marie rencontrera maintenant Jésus comme il est vraiment, glorifié et ressuscité, est la communauté. Marie doit passer du régime prépascal au régime de Pâques. Sa proclamation aux autres disciples indique clairement qu'elle a effectivement fait cette transition. Elle ne parle plus du «Rabbouni ». Comme premier apôtre de la résurrection, elle proclame: «j'ai vu le Seigneur ${ }^{36}$ ».

Dans l'épisode de Thomas, les choses sont tout à fait différentes. L'expérience de Pâques a eu lieu. La communauté ecclésiale, constituée par la Nouvelle Alliance accomplie par Jésus la nuit de Pâques, proclame à Thomas: "Nous avons vu le Seigneur ». Marie était la première des témoins apostoliques de la communauté pascale. Thomas est le premier représentant de la génération postpascale qui doit répondre dans la foi à leur témoignage.

Thomas ne fait pas, comme Marie, que mal comprendre son expérience: il refuse catégoriquement de croire le témoignage de la communauté. Il dit en effet: «Vous pouvez avoir vu le Seigneur, mais je ne l'ai pas

36. Bien que la discussion sur ce point aille au-delà de la portée de cet essai, je veux noter le consensus croissant parmi les chercheurs et les théologiens bibliques, à savoir que Marie de Magdala est, par chaque critère disponible dans le Nouveau Testament, un apôtre. Perkins (1992) affirme que l'épisode johannique de Marie de Magdala établit au moins une femme comme témoin indépendant de la résurrection. Okure (1992, 184-185) remarque correctement que Marie de Magdala n'est pas simplement "l'apôtre aux apôtres", comme si sa mission finissait quand la leur commence. Elle est l'apôtre chargé d'annoncer la résurrection à l'Église. Cependant, son rôle n'est pas limité à annoncer la résurrection (comme les disciples d'Emmaüs), mais elle est chargée (comme Paul) de proclamer la bonne nouvelle, c'est-à-dire le nouveau statut des croyants comme enfants de Dieu. Lee (1995, 46-47) dit qu'elle est le premier apôtre, le premier disciple du Seigneur ressuscité et la représentante de la communauté de foi. Une étude exégétique/théologique importante de tout le matériel du Nouveau Testament sur Marie de Magdala et qui arrive pratiquement à la même conclusion est celle de G. O'Collins et D. Kendall (1987). Le consensus croissant au sujet de l'identité apostolique de Marie de Magdala fait appel principalement au texte johannique. 
vu et jusqu'à ce que je le voie physiquement, je ne croirai pas. » Thomas ne nie pas leur expérience. Il dit simplement qu'il ne substituera pas leur expérience à la sienne. En quoi consiste sa mécompréhension ? Ce n'est pas leur expérience qu'il doit accepter en lieu et place de la sienne, mais c'est sur leur témoignage que sa propre expérience doit être fondée. C'est le problème de tous les croyants à travers les siècles qui doivent saisir d'une manière ou d'une autre que la foi n'accepte pas quelque chose comme vrai sur la base d'une autorité externe. Il faut permettre au témoignage de l'Église de devenir une expérience personnelle, par l'action de l'Esprit du Dieu vivant présent en Jésus. Il est important de noter que l'attitude de Thomas aurait été aussi problématique durant le temps du Jésus prépascal qu'elle l'était après Pâques. L'expérience sensorielle joue un rôle important dans la foi, mais non comme une preuve physique «des faits ». On pourrait manger du pain au lac de Tibériade et, précisément comme Jésus l'a dit aux foules qui l'ont cherché, ne pas voir le signe de Jésus comme pain de vie (voir 6,26), mais chercher une garantie sûre de nourriture matérielle. Les pharisiens, au chapitre 9, ont vu l'homme aveugle guéri et les Juifs, au chapitre 11, ont vu Lazare appelé hors du tombeau après quatre jours. Donc, on pourrait sonder les blessures de Jésus ressuscité et ne pas voir le signe de sa présence réelle. De manière similaire, dans le temps postpascal, Jésus est disponible — que ce soit dans l'Eucharistie, l'Écriture ou l'expérience mystique seulement au regard la foi.

Jésus, qui est déjà "là »- puisqu'il sait ce que Thomas a dit — apparaît à la communauté de laquelle Thomas avait été séparé, non seulement physiquement, mais aussi spirituellement. L'être de Jésus, sa présence réelle, est précisément ce que la communauté transmet à Thomas par son témoignage. La communauté ne rapporte pas un événement passé que Thomas aurait manqué par hasard et qu'il devrait maintenant accepter par les mots de quelqu'un d'autre. La communauté témoigne d'une réalité présente disponible à lui dans la foi, comme elle l'est à la communauté dans la foi. La communauté lui indique cette réalité. Le commandement de Jésus à Thomas est: "Ne sois pas incrédule, mais croyant ». L'invitation à toucher, comme nous l'avons vu, n'est pas une invitation à la vérification physique (qui ne peut pas causer ou fonder la foi), mais une invitation à l'expérience sacramentelle: voir ce que la crucifixion signifie vraiment, s'approprier ce que le côté ouvert offre vraiment.

Une expérience sacramentelle n'est pas désincarnée. C'est précisément une expérience du spirituel au sein du matériel. Jésus invite tous ses disciples d'après Pâques à une expérience qui est en continuité avec la foi fondée sur 
les signes exécutés dans l'expérience prépascale, et en même temps différente de cette foi. La continuité consiste en la médiation matérielle, l'effective expérience sensible du voir, de l'entendre, du goûter et du toucher. Pourtant, le matériel de médiation n'est plus le pain périssable du lac de Tibériade, mais le repas eucharistique coulant du côté ouvert de Jésus glorifié; ce n'est plus la vue physique restituée dans les eaux de Siloé, mais l'ouverture baptismale des yeux de la foi dans l'eau coulant de cette même source. Thomas signifie sa conversion dans son exclamation: "Mon Seigneur et mon Dieu ", qui est une réponse non à une preuve charnelle, mais à ce que Thomas ne pouvait pas voir physiquement, mais seulement dans la foi. Lui et tous les disciples après lui viennent à la foi par une expérience de signes, médiations matérielles d'une réalité spirituelle. Mais les signes dans ce nouveau régime ne sont pas la chair visible du Jésus prépascal, mais le corps sacramentel du Seigneur qui est l'Église et qui est médiatisé par elle.

Toute la deuxième moitié du récit de résurrection johannique est un déploiement du nouveau régime des signes qui supplanteront les signes du régime prépascal. Le signe fondamental, le sacrement originaire (Ursakrament) de Jésus réellement présent, est la communauté ecclésiale elle-même qui est maintenant le corps de Christ, le Nouveau Temple élevé dans le monde. La communauté témoigne de la parole proclamée enracinée dans l'Écriture, à travers la célébration des sacrements, son ministère de réconciliation, sa communauté d'amour mutuel qui lave les pieds et établit la vie, et la cohabitation en Jésus de ses membres dans la prière contemplative. La réponse à ce témoignage, des premiers disciples jusqu'à la suite des âges, est la reconnaissance de Jésus comme Seigneur et Dieu, reconnaissance à laquelle il réplique: "Bénis êtes-vous... parce que vous croyez ».

Le but de ces deux épisodes sur le «toucher» est d'aider le lecteur à faire les mêmes transitions que Marie de Magdala et Thomas le Jumeau ont dû faire: d'une fantaisie romantique de contemporanéité avec le Jésus prépascal, en passant par l'expérience pascale de la mort et de la vie nouvelle, jusqu'à la foi dans le Seigneur glorifié et ressuscité. Mais en faisant cette transition, deux extrêmes doivent être évités. L'un est de voir l'Église non comme une médiation de Jésus ressuscité lui-même, mais comme un substitut exhaustif pour un Jésus qui n'existe plus. L'autre est une tentative gnostique de se relier à Jésus dans une spiritualité "Jésus-et-moi » purement spirituelle, qui rejette la structure sacramentelle du corps ecclésial du Seigneur comme une organisation simplement humaine qui ne joue aucun rôle nécessaire ou essentiel dans notre rencontre avec Jésus. Marie de 
Magdala a dû prendre conscience que l'Église est le corps de Christ (Jésus n'est pas un cadavre) et Thomas a dû prendre conscience que l'Église est le corps de Jésus (non une substitution peu satisfaisante pour lui).

La communauté ecclésiale, en faisant dans le monde les œuvres que Jésus a faites (voir 14,12), est vraiment le corps du Christ, la "personne corporative» qui est l'organe de l'action salvifique de Jésus dans le monde. Mais cela peut seulement être le cas si Jésus lui-même, le principe de ce corps ecclésial, est vraiment vivant dans la pleine intégrité de son humanité personnelle. Voilà l'importance significative de maintenir que Jésus est ressuscité corporellement d'entre les morts. Ce que le corps signifie — identité numérique, subjectivité personnelle fondant la présence interpersonnelle et l'action efficace dans le monde - se vérifie en lui après sa mort sur la croix. Jésus n'est plus dans la chair: il n'est plus mortel. Il n'est plus soumis aux conditions du temps, de l'espace, de la causalité. La description du tombeau comme vide de son cadavre, sa non reconnaissance par Marie de Magdala, sa capacité à apparaître au milieu de ses disciples en dépit des portes fermées, sa connaissance de ce que Thomas a pensé et a dit en son absence, tout ceci constitue des dispositifs narratifs qui insistent tant sur la corporéité réelle que sur la condition non-charnelle de Jésus ressuscité.

Si Jésus n'est pas un sujet réel, distinct, personnel, une vraie personne corporelle, il n'y a aucun fondement ontologique pour la mystique chrétienne: la rencontre de Jésus a été un trait constant de la spiritualité de l'Église, au moins à partir d'Étienne qui, lors de sa lapidation, a vu Jésus debout à la droite de Dieu, et de Paul qui a appris que c'était Jésus qu'il persécutait - et jusqu'à notre époque. Si Jésus est simplement physiquement ressuscité, s'il est toujours dans la chair, alors il ne peut pas être médiatisé par une communauté dont il serait non seulement distinct, mais séparé.

En résumé, le récit de la résurrection de Jean ne traite pas du rétablissement et de la justification de Jésus après sa mort honteuse. La question est plutôt de savoir où et comment ses disciples rencontreront Jésus comme leur Seigneur et Dieu: la première génération étant symbolisée par Marie de Magdala, et tous ceux qui n'étaient pas avec cette première génération lorsque Jésus est venu étant symbolisés par Thomas le Jumeau.

Dans l'évangile lui-même, croire est présenté comme une réponse à la vue des œuvres de Jésus et à l'audition de son discours révélateur. Les chercheurs johanniques continuent de discuter pour savoir si l'évangile de Jean a été écrit pour rendre illégitime la foi fondée sur la vue des signes, au profit de la foi basée uniquement sur l'audition, ou pour présenter la vue et 
l'audition comme les médiations indispensables de la révélation ${ }^{37}$. Je crois que la nature même de l'incarnation comme symbolisation de la Sagesse/Parole de Dieu dans une forme sensible pointe vers la deuxième position. Le Verbe de Dieu est devenu chair; nous avons vu sa gloire; nos mains ont touché le Verbe de Vie. Et il est toujours avec nous.

Cependant, c'est précisément parce que Jésus est apparu dans la chair, c'est-à-dire comme un être humain mortel, que sa vie humaine comme chair devait se terminer. Mais si, comme je crois être le cas, la dynamique de l'expérience sensible qui médiatise la foi est un trait permanent de l'économie révélationnelle du salut, elle doit, d'une manière ou d'une autre, continuer après le départ du Jésus prépascal par la mort. Le chapitre 20 de l'évangile de Jean - éclairé par les derniers discours dans lesquels Jésus explique son "départ " par la mort comme un nouveau mode de "venue » à ses disciples dans l'Esprit (Jn 17-18) — est une tentative d'élucider la manière dont la dynamique de l'expérience sensorielle qui médiatise la foi est réalisée sous un autre mode après la résurrection. Les épisodes de Marie de Magdala et de Thomas le Jumeau explorent l'appropriation personnelle par les disciples du nouveau lieu et du nouveau mode d'expérience de Jésus, ressuscité corporellement et maintenant actif à travers son corps ecclésial. La catégorie de corps, non plus assimilée à celle de chair - un corps qui est matériel dans le sens d'un principe d'individuation, mais non dans le sens d'un principe physique - est utilisée par l'évangéliste pour assurer le lecteur d'une chose: c'est Jésus lui-même qui, non seulement est glorifié en présence de Dieu, mais est revenu vers nous. Nous le verrons, nous l'entendrons, nous le toucherons, nous ferons l'expérience de sa présence réelle dans nos vies et dans notre monde, par notre participation à la vie de la communauté ecclésiale. Mais nous sommes également assurés que nous sommes sa présence réelle, son corps en ce monde, individuellement et collectivement - une conviction qui constitue aussi un défi : être réellement sa présence, son corps. Jésus dit que «ce jour-là ", à savoir notre propre jour postpascal, "vous saurez que je suis en mon Père et vous en moi et moi en vous » $(\mathrm{Jn} 14,20)$ et que «celui qui croit en moi fera, lui aussi, les œuvres que je fais; et il en fera même de plus grandes » (Jn 14,12).

37. Pour une bonne vue d'ensemble de ce débat, voir Judge (1992). L'éventail des positions va de ceux qui considèrent l'évangile de Jean comme une critique et/ou un rejet de la foi basée sur des signes, en passant par ceux qui voient les signes comme jouant un rôle dans la foi seulement durant la vie prépascale de Jésus, jusqu'à ceux qui voient les signes comme importants de manière permanente pour la foi, quoique le genre de signes soit différent après la résurrection. Ma position appartient à la dernière catégorie. 


\section{Références}

Borg, M. (1994), Meeting Jesus Again for the First Time: The Historical Jesus and the Heart of Contemporary Faith, San Francisco, Harper.

Bracken, J.A. (2004), "The Body of Christ: An Intersubjective Interpretation ", Horizons, 31, p. 7-21.

Brown, R.E. (1970), The Gospel According to John XIII-XXI, Garden City, Doubleday (Anchor Bible 29A).

Bultmann, R. (1955), Theology of the New Testament, vol. 2 / trad. par K. Grobel, New York, Charles Scribner's Sons.

Byrne, B.J. (1985), "The Faith of the Beloved Disciple and the Community in John 20 ", Journal for the Study of the New Testament, 23, p. 83-97.

Clifford, R.J. (1990), «Exodus », dans R.E. Brown, J.A. FitzMYer et R.E. MurPhy, dir., The New Jerome Biblical Commentary, Englewood Cliffs, Prentice Hall, p. 44-60.

Coloe, M. (1999), "Like Father, Like Son: The Role of Abraham in Tabernacles. John 8:31-59», Pacifica, 12, p. 1-11.

Crotty, R. (1999), "The Two Magdalene Reports on the Risen Jesus in John 20 », Pacifica, 12, p. 156-168.

Cullmann, O. (1955-1956), «Immortality of the Soul or Resurrection of the Dead: The Witness of the New Testament ", Harvard Divinity School Bulletin, 21, p. 5-36.

DeConick, A.D. (1997), «»Blessed Are Those Who Have Not Seen» (Jn 20 :29) : Johannine Dramatization of an Early Christian Discourse ", dans J.D. Turner et A. MACGuire, dir., Nag Hammadi Library after Fifty Years. Proceedings of the 1995 Society of Biblical Literature Commemoration, Leiden, Brill (Nag Hammadi and Manichaean Studies 44), p. 381-398.

Dupont, L., C. LASH et G. Levesque (1973), « Recherche sur la structure de Jean 20 », Biblica, 54, p. 482-498.

ECKstein, H.-J. (2002), «Bodily Resurrection in Luke », dans T. Peters, R.J. Russell et M. Welker, dir., Resurrection: Theological and Scientific Assessments, Grand Rapids, Eerdmans, p. 115-123.

GoEDT, M. de (1962), «Un schème de révélation dans le quatrième Évangile », New Testament Studies, 8, p. 142-150. 
Green, B. (2003), "The Wisdom of Solomon and the Solomon of Wisdom: Tradition's Transpositions and Human Transformation ", Horizons, 30, p. 41-66.

Jonge, M. de (1978), "Signs and Works in the Fourth Gospel », dans T. BaArda, A.F.J. Klijn et W.C. VAn UnniK, dir., Miscellanea Neotestamentica 2, Leiden, Brill (Supplements to Novum Testamentum 48), p. 107-125.

Judge, P.J. (1992), «A Note on Jn 20 :29», dans F. van Segbroeck, C.M. Tuckett, G. van Belle et J. Verheyden, dir., Festschrift Frans Neirynck, vol. 3, Leuven, Leuven University Press, p. 2183-2192.

Lampe, P. (2002), "Paul's Concept of a Spiritual Body», dans T. Peters, R.J. Russell et M. WelKer, dir., Resurrection: Theological and Scientific Assessments, Grand Rapids, Eerdmans, p. 103-114.

La Potterie, I. de (1984), "Genèse de la foi pascale d'après Jn 20 ", New Testament Studies, 30, p. 26-49.

LEE, D.A. (1995), «Partnership in Easter Faith: The Role of Mary Magdalene and Thomas in John 20 ", Journal for the Study of the New Testament, 58, p. 37-49.

(2002), Flesh and Glory: Symbol, Gender, and Theology in the Gospel of John, New York, Crossroad.

MATERA, F.J. (1989), «John 20,1-18 », Interpretation, 43, p. 402-406.

McKenzIE, J.L. (1965), "Sheol », dans J.L. McKenZIE, dir., Dictionary of the Bible, Milwaukee, Bruce, p. 800-801.

MoINGT, J. (1972), "Immortalité de l'âme et/ou résurrection ", Lumière et Vie, 21, p. 65-78.

Mollat, D. (1974), «La foi pascale selon le chapitre 20 de l'Évangile de saint Jean ", dans E. DHANIS, dir., Resurrexit: Actes du symposium international sur la résurrection de Jésus, Rome, Libreria Editrice Vaticana (Essai de théologie biblique), p. 316-334.

Moloney, F. (1998), The Gospel of John, Collegeville, Liturgical Press (Sacra Pagina 4).

Murphy-O'Connor, J. (1990), "The First Letter to the Corinthians » dans R.E. Brown, J.A. FitZMyer et R.E. Murphy, dir., The New Jerome Biblical Commentary, Englewood Cliffs, Prentice Hall, p. 816-829.

NicholsburG, G.W. (1972), Resurrection, Immortality, and Eternal Life in Intertestamental Judaism, Cambridge, Harvard University Press (Harvard Theological Studies 26). 
O’Collins, G. et D. Kendall (1987), «Mary Magdalene as Major Witness to Jesus' Resurrection ", Theological Studies, 48/4, p. 631-646.

Okure, T. (1992), "The Significance Today of Jesus' Commission to Mary Magdalene ", International Review of Mission, 81, p. 177-188.

PERKINS, P. (1992), «»I Have Seen the Lord» (John 20,18) : Women Witnesses to the Resurrection ", Interpretation, 46, p. 31-41.

Rahner, K. (1966), "The Theology of the Symbol", dans Theological Investigations, vol. 4: More Recent Writings / trad. Par K. Smyth, Baltimore, Helicon Press, p. 221-252.

SCHNEIDERS, S.M. (1983), The Johannine Resurrection Narrative: An Exegetical and Theological Study of John 20 as a Synthesis of Johannine Spirituality, vol. 1, Ann Arbor, University Microfilms.

- $\left(2003^{2}\right)$ [1999], Written That You May Believe: Encountering Jesus in the Fourth Gospel, New York, Crossroad.

WARD, G. (1998), «Transcorporeality: The Ontological Scandal », Bulletin of the John Rylands University Library of Manchester, 80, p. 235-252. (1999), "Bodies: The Displaced Body of Jesus Christ », dans J. Milbank, C. Pickstock et G. Ward, dir., Radical Orthodoxy: A New Theology, Londres / New York, Routledge, p. 163-181.

WolfF, H.W. (1996) [1974], Anthropology of the Old Testament, Mifflintown, Sigler. 


\section{Résumé}

Cet article s’intéresse au rôle que joue la corporéité de Jésus dans sa relation avec les disciples après la résurrection. À travers l'étude de Jn 20, notamment les commandements contradictoires que Jésus adresse à Marie de Magdala (20,11-18) et à Thomas (20,24-29), il propose de comprendre que la résurrection n'est pas physique mais qu'elle est corporelle. Le corps du Ressuscité continuerait ainsi de médiatiser sa relation aux disciples, d'une façon qui est à la fois en continuité et en discontinuité avec la manière dont il le faisait durant sa carrière prépascale.

\section{Abstract}

This paper focuses on the role played by Jesus' corporality in his relationship to the disciples after the resurrection. Through the study of Jn 20, especially the conflicting commandments given to Mary Magdalene (20,11-18) and Thomas (20,24-29), it is suggested that the resurrection is not to be understood physically, though it is bodily. Thus, the body of the Resurrected is still mediating his relationship with his disciples, but in a way that is both continuous and discontinuous with the way it did in his pre-Easter career. 